\title{
INVESTIGACIONES
}

\section{Mejora educativa a partir de asesoría externa: el complejo camino hacia la sostenibilidad*}

\author{
Educational improvement through external consultancy: \\ The difficult road towards sustainability

\section{Melhoramento da educação através de assessoria externa: o complexo caminho à sustentabilidade}

\author{
Daniel Ríos, ${ }^{a}$ Paula Villalobos ${ }^{b}$ \\ aDepartamento de Educación. Universidad de Santiago de Chile \\ Telf.: (56)27184653. Correo electrónico: daniel.rios@usach.cl \\ bepartamento de Educación. Universidad de Santiago de Chile \\ Telf.: (56)27184652. Correo electrónico: paula.villalobos@usach.cl
}

\section{RESUMEN}

El presente artículo presenta un estudio que en una primera fase buscó relevar los logros que se han sostenido en cuatro liceos municipales, que recibieron asesoría técnica, desde los significados de los actores escolares luego de tres años de finalizado dicho asesoramiento. Como segunda etapa, se incluye el seguimiento realizado un año después a la institución que presentó sostenibilidad de la mejora. Se trata de un estudio cualitativo, que utilizó entrevistas y grupos de discusión a directivos y docentes. Los resultados muestran que en la instituciones donde se visualiza sostenibilidad de la mejora, es clave es el liderazgo directivo, la autonomía, la sistematización de la labor, el clima laboral positivo y el trabajo colaborativo. La falta de recursos, la despreocupación por la sucesión del liderazgo, el clima negativo, la escasa apropiación de la propuesta de la asesoría y las expectativas negativas sobre los estudiantes, representaron obstaculizadores de una mejora sostenible.

Palabras clave: sostenibilidad, mejora educativa, asesoría externa, condiciones institucionales, innovación educativa.

\begin{abstract}
This article shows a study which, firstly, attempts to reveal the achievements of four public high-schools receiving technical consultancy from the perception of school actors, three years after the assistance took place. Secondly, it includes the monitoring made a year later to the institution presenting improvement sustainability. This is a qualitative study which used interviews and focus groups with teachers and directive teams. Results show that institutions showing a sustainable improvement had directive leadership, autonomy, systematization of work, positive work environment and collaborative work as the key of their success. The lack of resources, the negative work environment, the indifference of leadership succession, the lack of commitment with the proposal, and the negative expectation on the students represented hinders for a sustainable improvement.
\end{abstract}

Key words: sustainability, educational improvement, external assistance, institutional conditions, educational innovation.

El presente estudio fue realizado con el apoyo y financiamiento de un Proyecto DICYT (Dirección de Investigación Científica y Tecnológica). Dependiente de la Vicerrectoría de Investigación desarrollo e innovación de la Universidad de Santiago de Chile. 


\section{PLANTEAMIENTO DEL PROBLEMA}

Las políticas educativas tendientes a generar procesos de mejora educativa han impulsado desde 2002 a la fecha planes de asistencia técnica externas. Ejemplos de ello son las Escuelas Críticas en enseñanza básica, el Programa de Liceos Prioritarios en enseñanza media. Dichas asesorías estuvieron centradas en apoyar a instituciones con bajos resultados educativos y que atienden a una población altamente vulnerable desde el punto de vista psicosocial. A partir de 2008, con la ley de Subvención Escolar Preferencial, la asistencia técnica externa se convierte en la herramienta central de las políticas de mejoramiento educativo.

Dada la relevancia que actualmente ocupa esta modalidad de intervención, resulta necesario generar conocimiento sobre las características y efectos de la asistencia educativa sobre la institución escolar y cómo este proceso puede contribuir a un proceso de mejoramiento educativo que se vuelva sostenible en el tiempo. Los estudios nacionales sobre asistencia técnica externa a los establecimientos educacionales coinciden en que su mayor debilidad es la falta de sostenibilidad de las mejoras logradas (Bellei, Osses y Valenzuela, 2010; Marchant, Salin y Williams, 2009), existiendo el riesgo de que no se logre instalar capacidades nuevas en las escuelas asesoradas (Espínola y Silva, 2009). La evidencia sobre asistencia técnica externa a los establecimientos se limita a estudios sobre implementación y evaluación de resultados, sin abordar en profundidad el tema de la sostenibilidad (Espínola y Silva, 2009; Osses y Raczynsky, 2010).

La experiencia de Asistencia Técnica llevada a cabo por la Universidad de Santiago de Chile a 12 liceos que formaron parte del Programa Liceos Prioritarios, en convenio con el Ministerio de Educación entre 2006 y 2010, en cuatro regiones del país, muestra varios logros en los diferentes ámbitos de gestión asesorados, así como evidencia las condicionantes que favorecieron u obstaculizaron estas acciones (Ríos y Villalobos, 2012; Ríos et al., 2010; Villalobos, 2009, 2010¹). Concretamente, en la gestión con el sostenedor se valora la participación y el compromiso de este con la labor directiva. En el ámbito de la gestión directiva se valora la mayor organización y coordinación en torno a objetivos y la atención a evidencias de las acciones desarrolladas. También se destaca el fortalecimiento del liderazgo de la jefatura técnico pedagógico. En la convivencia escolar, en tanto, la revisión participativa de los manuales de convivencia y su enriquecimiento desde un énfasis formativo, así como el fortalecimiento del vínculo entre los liceos y redes externas de apoyo, corresponden a avances reconocidos desde las instituciones. En la dimensión pedagógica, se valora el fortalecimiento del trabajo colaborativo docente, la reflexión pedagógica y las acciones de mejoramiento en los procesos de diseño curricular de aula, didácticos y evaluativos. Se agrega el trabajo en aula de modelaje de propuestas metodológicas como el análisis del desempeño docente mediante la observación de clases.

Considerando los antecedentes presentados y las mejoras obtenidas con la asistencia técnica proporcionada por la USACH, la pregunta que orientó esta investigación, a modo de problema, es: ¿son sostenibles las mejoras resultantes de la asistencia técnica en liceos prioritarios, en qué condiciones y con qué procedimientos las mejoras logran sostenibilidad?

A partir de los resultados informados al finalizar la asistencia técnica de la USACH por los establecimientos de educación media en el marco del Programa Liceos Prioritarios,

Información basada en los informes de evaluación anuales de la Asistencia Técnica, encargados por el Equipo responsable de la asesoría a profesionales externos durante los años 2009 y 2010. 
indagaremos sobre la sostenibilidad de las mejoras logradas, luego de tres años de finalizada la asistencia técnica. Siguiendo una lógica inductiva, se espera producir conocimiento sobre los procedimientos y condiciones bajo los que una mejora se sostiene en el liceo, después de concluida la presencia de la institución asesora.

Más concretamente, este trabajo busca relevar los logros que se han sostenido en los liceos que recibieron la asesoría técnica e interpretar los factores asociados a esta sostenibilidad, desde los significados de los actores escolares.

\section{MARCO CONCEPTUAL}

La sostenibilidad de la mejora educativa posibilita que las instituciones educativas entren en un proceso de mejoramiento que se mantiene y profundiza en el tiempo, lo que supone adhesión y compromiso de toda la comunidad que la integra. En este sentido, Hargreaves y Fink (2006), plantean que una tarea central de los líderes escolares corresponde a sostener el aprendizaje. Dichos autores caracterizan este concepto aludiendo a su duración en el tiempo, el compromiso y su contribución al bien común. Resaltan la necesidad de apoyo, recursos, compromiso y generación de capacidades a largo plazo en los docentes.

Uno de los elementos que se asocian a la sostenibilidad corresponde al liderazgo distribuido, enfatizando con ello una visión de liderazgo que se aleja de las individualidades y forma parte de la cultura de la escuela, logrando promover en todos sus miembros, la iniciativa y la innovación (González, 2003 Cit. en Hernández y Santana, 2010; López, 2010; Murillo y Krichesky, 2012; Sepúlveda y Murillo, 2012).

Profundizando en el concepto, los autores complementan su definición a partir de una serie de cualidades. La profundidad en cuanto se debe preservar aquello que enriquece la vida, como el aprendizaje profundo y la atención y el cuidado hacia otros. La longitud, relacionada con su continuidad en el tiempo. La anchura aludiendo a la distribución del liderazgo. La justicia, que expresa su orientación hacia el beneficio de la comunidad y su entorno. La diversidad que se opone a la estandarización. Recursos en cuanto la sostenibilidad supone un incremento material y de recursos humanos. Y la conservación que alude al reconocimiento de lo realizado en el pasado (Hargreaves y Fink, 2006).

Considerando los conceptos anteriores, es posible afirmar que la sostenibilidad de la mejora educativa corresponde a un fin altamente relevante y deseable, por tanto resulta fundamental, generar conocimiento respecto de los elementos que facilitan y obstaculizan los procesos de cambio para alcanzar dicho estado.

Particularmente, este trabajo se refiere a los procesos de mejora educativa que son apoyados por asesorías externas, dado que para las escuelas con alumnos de bajo nivel socioeconómico y deficientes logros educacionales, las políticas educacionales han dado un rol crecientemente central a la provisión de servicios de asistencia técnica educativa. (González y Bellei, 2013). Junto a ello, se analizan los aportes de la investigación en torno a los elementos que aportan positiva y negativamente a la sostenibilidad de dicha mejora.

La evidencia sobre los programas de asistencia técnica educativa en Chile muestra que, en promedio y cuando han acumulado al menos cuatro años de trabajo en las escuelas, estos han tenido un impacto positivo y significativo en los logros académicos de los alumnos, tanto en lenguaje como en matemáticas, pero que dicho impacto tiende a desvanecerse rápidamente una vez concluida la intervención (Espínola et al., 2008; González y 
Bellei, 2013). A nivel latinoamericano, casi no existen estudios de sustentabilidad del mejoramiento escolar, y la investigación disponible refleja que, en muchos casos, cuando sucede dicho mejoramiento tiende a desaparecer a través del tiempo (Valenzuela y Allende, 2014), dejando en evidencia que los procesos de mejoramiento no son lineales (Creemers, et al., 2007; González y Bellei, 2013). Aún más, las escuelas tendrían enormes dificultades para alterar su nivel de desempeño original (Valenzuela y Allende, 2014), especialmente las menos efectivas. Asimismo, Razcynski y Muñoz (2006 Cit. en Valenzuela y Allende, 2014) indican que el mejoramiento alcanzando por estos programas de apoyo entre las escuelas más vulnerables es altamente inestable.

\subsection{FACTORES QUE ATENTAN CONTRA LA SOSTENIBILIDAD DE LA MEJORA}

Adentrándonos en aquellos fenómenos que obstaculizan el logro de mejoras sostenibles, se encuentra una visión pesimista sobre las posibilidades de éxito escolar de los estudiantes, justificada por la pobreza de sus familias y del entorno. Esta percepción negativa incluye dudas acerca de las familias y su capacidad para formar, motivar y apoyar a los alumnos (González y Bellei, 2013; Hernández, Murillo y Martínez, 2014).

Por otra parte y frente a la acción de un proceso de asesoría, el nivel de involucramiento de la comunidad se reduce a individualidades. Se observa un proceso de cambios parcelado, que no afecta a todas las áreas relevantes ni involucra a una masa crítica de docentes; focalizado principalmente en tácticas para aumentar los puntajes en la evaluación externa estandarizada; basado en procesos frágiles e inestables de transformación, fuertemente dependientes de algunas pocas personas aisladas. Esta forma de "mejoramiento escolar" no sustantivo, no lograría penetrar en la cultura ni en la estructura de los establecimientos (González y Bellei, 2013). De esta forma, la evidencia empírica da cuenta de que en instituciones estancadas desde el punto de vista de la mejora, los miembros de la comunidad escolar nunca llegan a apropiarse de las iniciativas de innovación (Sepúlveda y Murillo, 2012).

En contraste, en las escuelas con "mejoramiento escolar sostenido" se observan procesos de cambio multidimensionales, que logran afectar las diversas áreas de trabajo de la escuela y de la docencia en particular; impulsados por un trabajo colectivo basado en la participación sistemática de la gran mayoría de los docentes y en colaboración con los directivos, y expresados en una transformación profunda de los modos de hacer y pensar profesionalmente. Este mejoramiento escolar genuino logra modificar la visión de los actores y se institucionaliza (González y Bellei, 2013; López, 2010; Sepúlveda y Murillo, 2012).

Las instituciones estancadas suelen haber experimentado experiencias de proyectos sucesivos con una historia de fracasos, que son vistos como una imposición ya sea externa a la escuela o como una demanda de la dirección (Sepúlveda y Murillo, 2012). Tampoco se evidencia una preocupación por proyectar en el tiempo los eventuales aprendizajes aportados por la asesoría, lo que estaría detrás de la rotación tanto de directivos como profesores (González y Bellei, 2013). Otro elemento obstaculizador de la sostenibilidad corresponde al clima organizacional negativo, caracterizado por conflictos no resueltos o inadecuadamente enfrentados (González y Bellei, 2013; Hernández et al., 2013).

Directamente ligado a los problemas de relación entre los docentes, en las escuelas menos eficaces se detecta una escasa implicación y compromiso de muchos profesores 
y profesoras (Hernández et al., 2013). Estos mismos autores logran puntualizar aquellos elementos propios de las escuelas menos eficaces incluyendo, entre otros, un clima de la escuela caracterizado por la desconfianza, la división, la desmotivación de los estudiantes, la falta de compromiso de los docentes, una dirección poco comprometida, que adolece de liderazgo; baja participación de la familia y bajas expectativas de logros (Hernández et al., 2013).

\subsection{FACTORES QUE CONTRIBUYEN A LA SOSTENIBILIDAD DE LA MEJORA}

En primer término, podemos mencionar la relevancia de las condiciones internas de organización de las escuelas, dentro de ellas el contar con directores y jefes técnicos que ejercen un rol activo en relación a los procesos de cambio. De hecho, se sabe que los países con mejores resultados tienen sólidos equipos directivos que guían los procesos de cambio en las escuelas (López, 2010; Muñoz y Marfán, 2011; Murillo y Krichesky, 2012). Dichos Equipos son capaces de ir generando un clima de buena convivencia y compromiso con la labor a realizar, haciendo que una parte mayoritaria de los actores del establecimiento comparta los propósitos del cambio, se involucren en los nuevos desafíos y cumplan las exigencias que el trabajo de asesoría requiere, dando apoyo permanente a los docentes, garantizando los tiempos y espacios de trabajo e involucrando en el cambio a todos o la mayoría de los docentes (González y Bellei, 2013; Muñoz y Marfán, 2011; Sepúlveda y Murillo, 2012).

Además de lo recién descrito, en las escuelas que logran dar continuidad a las mejoras se siguen utilizando las herramientas adquiridas durante la asesoría una vez concluida esta, dejando registro y sistematizando su trabajo (lo que facilita la transmisión ante la llegada de nuevos integrantes), apoyando y monitoreando el trabajo pedagógico, e implementando medidas de inducción para socializar y formar en este modo de trabajo a los nuevos docentes (González y Bellei, 2013; Murillo y Krichesky, 2012).

Respecto de las innovaciones pedagógicas, es clara la importancia que tiene el hecho de que los docentes visualicen el potencial de las nuevas metodologías, como condición para apropiarse de ellas y adoptarlas en su práctica regular. En dicha apropiación debe estar presente la generación de capacidades autónomas de trabajo en los profesores, enfatizando el uso de talleres prácticos, la discusión colectiva, el modelamiento y la elaboración propia de materiales (Anderson y Carrasco, 2013; González y Bellei, 2013; Murillo y Krichesky, 2012).

Dentro de las condiciones institucionales, también se cuenta la prioridad concedida al establecimiento de un clima escolar satisfactorio. Autores como López (2010) y Murillo y Krichesky (2012) señalan que en procesos de mejora sostenida se logra avanzar hacia la creación de una cultura profesional de aprendizaje, es decir, se crea una cultura que estimula el aprendizaje y el desarrollo profesional continuo, en el marco de una estructura en la cual tenga lugar ese desarrollo a través de la colaboración y el apoyo mutuo.

Unida a estas condiciones institucionales, para generar la sostenibilidad se requieren asesores capaces de ser creíbles para los docentes, evidenciar sus competencias en el área que asesoran y contar con experiencias de trabajo previos en contextos similares a los de la institución asesorada. Junto con las competencias profesionales, los asesores de las experiencias exitosas de asesoría muestran un compromiso y preocupación personal frente al trabajo, más allá del cumplimiento formal de las exigencias. También son capaces de desarrollar una comunicación fluida y cercana con los docentes y directivos. Se preocupan por conocer continuamente la 
percepción de los miembros de la escuela frente al proceso que se lleva a cabo, e identifican e intentar resolver las dificultades percibidas por los docentes (González y Bellei, 2013).

López (2010) enfatiza las condiciones institucionales como base fundamental sobre la cual los cambios son construidos cuando estos logran mantenerse en el tiempo, dichos elementos tendrían un carácter informal, como la cultura organizativa, los patrones y las redes informales de poder, la distribución del liderazgo o las pautas de comunicación. Particularmente, el autor hace mención a aspectos presentes en los profesores como el valor que representa para ellos enseñar en circunstancias difíciles, su compromiso con el desarrollo social de la comunidad, su cohesión y la creación de un fuerte sentido identitario.

Considerando lo anterior, las condiciones internas de la institución escolar resultarían sustantivas para el mejoramiento escolar en el largo plazo (González y Bellei, 2013; López, 2010; Sepúlveda y Murillo, 2012; Valenzuela y Allende, 2014).

En suma, este artículo aborda la sostenibilidad de la mejora educativa, la cual permite que las instituciones entren en un proceso de mejoramiento que se mantiene y fortalece en el tiempo. En particular, se estudia la sostenibilidad asociada a experiencias de asesoría externa educativa. Teniendo presente que tanto el liderazgo distribuido, el involucramiento de la comunidad, el compromiso de la comunidad, el monitoreo al trabajo pedagógico, la generación de una comunidad de aprendizaje docente y un sentido del beneficio comunitario son aspectos que parecen facilitar la sostenibilidad y, en oposición a ello, los procesos de mejora centrados en individualidades, con escasa participación de la comunidad, con climas organizacionales negativos y donde predominan entre los docentes visiones pesimistas acerca de las posibilidades de aprendizaje de los estudiantes, representan obstáculos para alcanzar logros sostenibles.

\section{DISEÑO METODOLÓGICO}

Se asume una aproximación cualitativa, en tanto esta perspectiva de análisis permite rescatar la forma en que los actores analizan la sostenibilidad de las acciones implementadas a partir de las ATES $^{2}$ en sus propios términos. Se adopta el paradigma interpretativo, fundamentalmente porque este promueve la comprensión e interpretación de la realidad desde los significados de las personas implicadas en los contextos educativos, estudiando sus creencias, intenciones, motivaciones y otras características del proceso educativo, los cuales no son observables directamente ni susceptibles de experimentación (Latorre, Rincón y Arnal, 1996). Por otro lado, formulamos un diseño emergente e interactivo que combina la utilización de entrevistas individuales y grupos de discusión.

\subsection{SELECCIÓN DE LOS ESTABLECIMIENTOS}

El tipo de muestreo es intencional, considerando como criterio esencial la inclusión de cuatro liceos pertenecientes a tres regiones del país (una del norte, otra del centro sur y una del extremo sur del país), que recibieron asistencia técnica y que de acuerdo a los informes anuales de evaluación presentaron logros o avances en la gestión directiva, gestión pedagógica, gestión de la convivencia escolar y gestión con el sostenedor.

Asistencia Técnica Educativa. Organismo acreditado ante el Ministerio de Educación de Chile para asesorar a establecimientos educacionales públicos en el ámbito de la gestión directiva, gestión pedagógica y gestión de la convivencia escolar. 
Un año después se continuó la recolección de información en uno de los cuatro liceos, que según la primera etapa del estudio, presentó sostenibilidad en las mejoras resultantes del proceso de asesoría.

Los participantes en los grupos de discusión, durante la primera etapa del estudio, fueron docentes de los liceos seleccionados. La selección de los participantes se realizó considerando profesores que fueron participantes activos de las ATE. Por otro lado, las entrevistas se realizaron a los miembros de los Equipos Directivos de cada establecimiento seleccionado.

Tabla 1. Participantes de primera etapa

\begin{tabular}{|c|c|c|c|c|}
\hline Participantes & Liceo 1 & Liceo 2 & Liceo 3 & Liceo 4 \\
\hline Director & 1 & 1 & 1 & 1 \\
\hline Jefe de $\mathrm{UTP}^{3}$ & 1 & 1 & 1 & 1 \\
\hline Orientador & 1 & 1 & 1 & 0 \\
\hline Inspector General & 1 & 0 & 0 & 1 \\
\hline Profesor de matemática & 3 & 3 & 3 & 2 \\
\hline Profesor de lenguaje & 3 & 3 & 3 & 1 \\
\hline Profesor de especialidad EMTP ${ }^{4}$ & & & & 1 \\
\hline Total: 35 & 10 & 9 & 9 & 7 \\
\hline
\end{tabular}

En la segunda etapa del estudio, participaron miembros del equipo de gestión y profesores de asignatura del liceo que presentó sostenibilidad de la mejora, según lo muestra la siguiente tabla.

Tabla 2. Participantes de segunda etapa

\begin{tabular}{ll}
\hline \multicolumn{2}{c}{ Participantes } \\
\hline Inspector General & 1 \\
Jefe de UTP & 1 \\
Orientadora & 1 \\
Docentes apoyo UTP & 2 \\
Profesores de asignatura & 4 \\
\hline Total & 9 \\
\hline
\end{tabular}

Encargado de los procesos técnico-pedagógicos en la institución escolar.

4 Profesor de Enseñanza Media Técnico Profesional. 
En una primera etapa, la recolección de datos se realizó a través de entrevistas individuales semi estructuradas y de grupos de discusión. Tanto los guiones de las entrevistas como los de los grupos de discusión, tuvieron como base los aspectos que fueron señalados como logros generados a partir de la asistencia técnica desde la visión de los docentes y de los directivos involucrados en ella. Para establecer las pautas guías, se tuvo como guía los informes de evaluación realizados por la Universidad y los informes presentados al MINEDUC una vez finalizado el proceso de asesoría externa. Las entrevistas tuvieron una duración de una hora aproximadamente.

En una segunda etapa se realizaron entrevistas semiestructuradas cuyas pautas guías se relacionaban con los logros que fueron sostenibles en el tiempo de acuerdo a la visión de los miembros de la comunidad educativa. El proceso general de análisis de los datos textuales se basó en los lineamientos propuestos por Rodríguez, Gil y García (1994), a partir de los supuestos planteados por Miles y Huberman (1994) quienes presentan el esquema de análisis como un proceso recursivo. La reducción de datos consideró, por un lado, el análisis de los datos textuales así como las notas de campo y, por otro, la formación de categorías analíticas tomando como base las categorías elaboradas durante el análisis de los grupos de discusión. Posteriormente, la fase de estructuración y organización de la información se realizó a partir de la elaboración de una guía para la codificación, la cual se fue modificando y refinando en el mismo proceso.

\section{RESULTADOS}

A continuación se presentan los resultados obtenidos tras el estudio. Organizados de acuerdo a los elementos que desde la visión de los actores pertenecientes a las distintas instituciones se mantienen o no en la institución como un logro del proceso de la asesoría. En este sentido, cabe destacar el alto grado de acuerdo que se da entre instituciones respecto de las condiciones asociadas negativamente a la sostenibilidad de la mejora. Cabe señalar que todos los aspectos asociados positivamente a la sostenibilidad de la mejora corresponden a una sola institución.

\subsection{ELEMENTOS ASOCIADOS NEGATIVAMENTE A LA SOSTENIBILIDAD, SEGÚN ÁREA DE GESTIÓN}

\subsubsection{Gestión con el sostenedor}

Un primer elemento que se opone a la sostenibilidad se relaciona con la falta de recursos. Los entrevistados señalan que les preocupa la situación financiera del sostenedor municipal, existiendo el temor de que rebajen las horas de contrato de los docentes, lo que impediría consolidar un trabajo colaborativo. Por otra parte, se hace visible la incertidumbre frente a quienes asumirán la dirección de la institución, donde ellos no tienen incidencia. Se considera una decisión política en manos del alcalde. Por tanto se manifiesta a nivel local, la falta de mecanismos que aseguren la sucesión del liderazgo, lo cual se opone a la sostenibilidad.

Otro aspecto relevado se relaciona con las horas contratadas para la labor del profesor jefe. Los contratos no contemplan tiempo de trabajo con estudiantes y apoderados. Las condiciones laborales de los docentes representan una limitación estructural para su labor, 
afectando directamente la posibilidad de contar con espacios para el trabajo docente conjunto, lo cual se opone a la sostenibilidad de la mejora educativa.

\subsubsection{Gestión pedagógica}

En la misma línea, refiriéndonos a elementos de continuidad de la labor de la asesoría, se plantea que se mantiene la evaluación de los avances de los aprendizajes, pero falta que estos datos sean mayormente analizados para convertirse en información relevante para los profesores. Al carecer de análisis y reflexión, esta labor se convierte en una acción burocrática que se opone a la sostenibilidad de la mejora. Estos resultados, un año más tarde, no presentan una variación significativa.

Otro elemento evidenciado en los resultados corresponde a las bajas expectativas de los docentes en torno al logro de aprendizaje de los estudiantes, lo que corresponde a un elemento asociado negativamente a la sostenibilidad de la mejora. También, la postura frente al aprendizaje de los estudiantes es variada, están aquellos que deciden aprobar a todos los estudiantes para disminuir su trabajo y no ser presionados por las autoridades. Otros docentes expresan una real preocupación por el aprendizaje que sus estudiantes alcanzan y hay otros que se enfocan a intervenir sobre las problemáticas de índole personal y familiar que presentan los estudiantes. Frente a estas visiones no hay consenso entre los profesores ni a nivel del sistema escolar, el que normativamente sería poco consistente, exigiendo acogida e inclusión, lo que para los profesores entra en contradicción con la exigencia por rendimiento académico. Un ejemplo de ello es la imposibilidad de dejar repitiendo a un estudiante por inasistencias, la imposibilidad de obligar a un apoderado a asistir a instancias formales de comunicación con los profesores.

Vinculado a lo anterior, emerge con fuerza de parte de los docentes una visión negativa sobre las familias. En alusión a los apoderados, se plantea que no todos participan del proceso, lo cual se atribuye a la gestión del profesor jefe. Hay deslegitimación del centro de padres debido al mal manejo de sus recursos. En este sentido, observamos la baja participación comunitaria, lo cual se asocia negativamente con la sostenibilidad de mejora. Los docentes observan dificultades en las familias de sus estudiantes, que se expresan en el vínculo padres e hijos, lo que limita la influencia positiva que pudiera esperarse de parte de los padres en torno al proceso educativo de sus hijos. Refieren permisividad, falta de límites y violencia intrafamiliar. En este sentido, la comunicación con los padres de los estudiantes tiene efectos positivos en un porcentaje menor de estudiantes. La realidad psicosocial de los estudiantes representaría, a su juicio, un fuerte desgaste para los docentes.

En relación al apoyo de otros profesionales, se valora el aporte y el apoyo profesional, pero se explicitan las implicancias de usar la evaluación diferenciada en estudiantes de enseñanza media, evidenciando desacuerdo respecto de su conveniencia para estimular el aprendizaje y la motivación de los estudiantes. En general, los profesores se verían obligados a hacer evaluación diferenciada a estudiantes que no lo requieren o que derechamente se sienten menoscabados por ese hecho frente a sus pares.

Se percibe escasa comunicación y coordinación del equipo de profesionales de apoyo con los docentes, escaso número de profesionales para responder a la demanda presente en los estudiantes, quienes llegan con una serie de dificultades de aprendizaje y retraso pedagógico; promedios que no reflejan su nivel de aprendizaje. A esto se agregan muchas dificultades en el ámbito de la comprensión lectora. 
Si bien la institución presenta este elemento de continuidad, el cual corresponde al abordaje de situaciones propias de los estudiantes con necesidades educativas especiales y con rezago pedagógico, se hace evidente un fortalecimiento de este ámbito de acción a través de una mayor reflexión sobre la oferta educativa que resulta más pertinente para estos estudiantes, lo que supone poder intensificar el diálogo entre los docentes y profesionales de apoyo. Dicha necesidad de acercamiento entre profesionales de apoyo y docentes es una necesidad que persiste un año después.

\subsubsection{Desmotivación de los estudiantes}

Los docentes sostienen que adolecen de recursos didácticos que permitan revertir la actitud de desmotivación de los estudiantes en clases, la que se explicaría por problemáticas de carácter familiar importantes. Otro elemento que resta efectividad a las clases está representado por la tecnología, la que a su juicio constituye un gran distractor.

Una problemática importante corresponde a la alta inasistencia a clases, lo que estaría íntimamente asociado a un bajo rendimiento. Además se considera problemática la sobrevaloración de la calificación en desmedro de la posibilidad de aprendizaje. A juicio de los docentes, el principal factor motivador es un factor externo de control, representado en la nota, lo que hace que las actividades de aprendizaje deban ser evaluadas siempre para asegurar su realización de parte de los estudiantes. Estos, además, adolecen de proyecciones futuras. Finalmente, en este plano se plantea que se daría una fuerte tendencia a responsabilizar a los docentes frente a los resultados de aprendizaje y nunca a los estudiantes, situación que se considera injusta.

\subsubsection{Gestión de la convivencia}

La asesoría aportó en la elaboración de un manual de convivencia y en la elaboración de protocolos de derivación de estudiantes, aspectos que se visualizan como logros, sin embargo su implementación se ve limitada por que ello supone el trabajo en equipo, que en la institución es débil. Una situación similar se vive en el tema del seguimiento de los estudiantes. No es oportuna la derivación de estudiantes, lo que incide en la deserción de estos. Los aspectos normativos se aplicarían parcialmente, quedándose en un nivel burocrático, opuesto a la sostenibilidad.

Otro elemento común al que se alude es la existencia de un clima organizacional negativo. Se plantea que existen bandos donde prevalecen intereses personales por sobre los institucionales, un alto nivel de desconfianza y división entre profesores. Todo lo cual es propio de instituciones que no logran mejoras sostenibles.

En el liceo estudiado en la segunda etapa, se visualiza una convivencia escolar positiva, tanto entre estudiantes, como en la relación de estos con los docentes. No obstante, de parte de los docentes se reconoce la necesidad de que la comunidad educativa en su conjunto pueda aplicar de manera más consistente las normas establecidas, y por otra parte fortalecer la reflexión en torno a la formación de los estudiantes más allá de sancionar faltas disciplinarias.

\subsubsection{Gestión directiva}

El trabajo en torno a metas y objetivos no fue adecuadamente socializado con los docentes, quienes hablan de falta de claridad en el fin de la asesoría. Los acuerdos no 
se aplican y no se realizan adecuados seguimientos de las acciones. La planificación, el monitoreo y evaluación, propios de instituciones donde se da la sostenibilidad de la mejora, son aspectos ausentes en tres de las cuatro instituciones estudiadas.

Si bien se valoran algunos aportes de la ATE (material e instrumentos de evaluación), lo común es que luego de terminada la asesoría no se continuó el trabajo. Este punto evidencia la escasa apropiación de la propuesta de la asesoría, el involucramiento individual y no colectivo con la asesoría y el bajo compromiso de los docentes con las innovaciones, a lo que se agrega una implementación desprovista de elementos que incidan en la renovación de las prácticas docentes en aula.

Otro elemento que se manifiesta de manera compartida es la ausencia de trabajo colaborativo. En este sentido, se plantea que el trabajo colaborativo docente es escaso, solo se da en algunas asignaturas. El trabajo docente sería predominantemente aislado, reduciéndose a la acción de planificación de clases, siendo también dificultoso trabajar como departamento. El escaso trabajo en equipo es propio de instituciones que no presentan mejoras sostenibles.

\subsection{ELEMENTOS ASOCIADOS POSITIVAMENTE A LA SOSTENIBILIDAD, SEGÚN ÁREA DE GESTIÓN}

\subsubsection{Gestión Directiva}

Es compartida la visión según la cual la asesoría permitió a la institución realizar un certero diagnóstico. Permitió sistematizar las acciones que ellos llevaban a cabo. A partir de aquello, se ha ido instalando la práctica de acompañamiento a los docentes, se han ido sistematizando procesos, que se expresan en protocolos de funcionamiento. Los equipos además mantienen encuentros semanales para evaluar y planificar su acción. Dichas cualidades de la gestión corresponden a elementos presentes en la gestión, de acuerdo a la visión de los entrevistados en la segunda etapa del estudio. Tanto la sistematicidad de la gestión, el monitoreo y la coordinación corresponden a elementos asociados positivamente con la sostenibilidad de la mejora.

Por otra parte, en cuanto a la participación docente se reconoce el haber tenido espacios de participación en la elaboración de proyectos de mejoramiento educativo; sin embargo, desde la visión del profesorado, la toma de decisiones sobre el producto final quedaría en manos del equipo directivo. De este modo, la participación de los docentes en torno al PME se habría centrado en el planteamiento de metas, no así en las estrategias para lograr dichas metas. A partir de esto, es posible afirmar que falta fortalecer espacios de participación más incidentes, considerando la mirada de los docentes.

Un año más tarde, mediando un cambio de Dirección, a juicio de los profesores su participación en la toma de decisiones pedagógicas se ha acrecentado, lo que se evidencia, entre otros, en la reformulación del PME, donde han sido incorporadas de manera importante las propuestas del profesorado.

En relación al espacio colaborativo, la posición de los directivos y de los docentes difiere. Mientras el equipo de gestión considera que hay tiempos disponibles y que se debe mejorar la organización de estos espacios, los docentes consideran que han perdido espacios de trabajo conjunto o bien que los que existen deben fortalecerse. Desde la opinión del Equipo Directivo, existe el tiempo, falta sistematizar estos encuentros; hay departamentos 
donde funciona este espacio y otros en que no sucede. Hace falta asignar tareas específicas, fortalecer liderazgos pedagógicos para contribuir a un diálogo profesional. Los docentes, en tanto, consideran que se ha instalado una mayor disponibilidad para trabajar en conjunto, que no existía en la institución al iniciarse la asesoría. El departamento de asignatura funcionaba en torno a tareas administrativas y no pedagógicas. De cualquier forma, esto constituye un aspecto a fortalecer en vías de la sostenibilidad. Se debiera profundizar en un diálogo profesional en torno a los caminos que resulten más pertinentes para el logro de los proyectos de innovación institucionales, visión que sigue presente, tanto en directivos como en profesores, un año más tarde. Respecto a este mismo tema, se evidencia la opción directiva por mantener horas de contrato frente al sostenedor, que permitan mantener las instancias de Consejo de Profesores y de Grupos Profesionales de Trabajo (GPT) lo que resulta relevante considerando que el trabajo colaborativo docente corresponde a un aspecto esencial en procesos de mejora sostenible.

\subsubsection{Gestión de la convivencia}

Tanto directivos como docentes destacan la cercanía y acogida y la positiva inserción en la comunidad. Se plantea que a nivel de equipo directivo, existe valoración y respeto mutuo. El equipo directivo, a su juicio, es cercano a los profesores y estudiantes, lo que aporta al clima positivo que repercute en la alta demanda de matrícula que presenta el liceo. Dicha situación sigue siendo considerada por los entrevistados como parte importante de las fortalezas de la institución. Por otra parte, cabe señalar que en la primera fase del estudio aparecía como una debilidad de este ámbito el hecho de que los docentes tendían a no plantear sus aprehensiones ni desacuerdos al equipo directivo. En la última etapa del estudio realizado, sin embargo, se evidencia una mayor capacidad de los docentes para plantear abiertamente sus posturas frente al equipo de gestión y lograr con ello incidir en la toma de decisiones, lo que ellos relacionan con la disposición del nuevo director del establecimiento.

\subsubsection{Gestión pedagógica}

Existe una pauta de observación de clases consensuada, que se aplica existiendo posteriormente una retroalimentación, lo cual se instaló a partir de la asesoría. La observación de clases, por tanto, constituye otro elemento de continuidad, sin embargo los docentes esperarían un mayor aporte didáctico en la fase de retroalimentación, ya que el análisis posterior se centraría solo en el cumplimiento de la estructura de la clase, lo cual ya está asumido por ellos.

Es posible observar entonces en esta acción, un potencial de monitoreo, planificación y evaluación, aspectos que se condicen con la sostenibilidad de la mejora educativa, no obstante falta profundizar en aquellos aspectos que de acuerdo a los docentes, resultarían más incidentes en la mejora de sus prácticas. En relación a este punto y ligado a lo expuesto en el párrafo anterior, los docentes plantean que durante el último año han podido consensuar con el equipo de gestión una pautas de observación más pertinentes a su realidad, así como los criterios y condiciones para que este proceso se lleve a cabo y verdaderamente incida en la mejora de la praxis docente.

Finalmente, se destaca una mayor inducción de los docentes que ingresan a la institución, así como un acompañamiento durante sus primeras clases, que tampoco 
era parte de la rutina institucional previa a la asesoría. Este aspecto podría contribuir al compromiso docente con la institución, con la adhesión a la misión, lo cual contribuye a la sostenibilidad de la mejora.

\section{DISCUSIÓN}

A partir de la primera y segunda etapa de este estudio, se han logrado establecer aspectos que han sido valorados positivamente a partir de la asesoría, y que además se asocian a procesos sostenibles de mejora. Estos logran permanecer y profundizarse, como aquellos relacionados con la sistematización, planificación y monitoreo de la labor desarrollada en la institución. Sin duda, esto brinda la posibilidad de valorar el grado de avance y la gestión en términos amplios.

Por otra parte y asumiendo la frecuente rotación de docentes, las acciones de inducción posibilitan dar continuidad a una forma de trabajo y facilitan la adhesión de los docentes a un proyecto común.

El clima organizacional, en tanto, se valora positivamente y ha progresado al contar con mayores espacios de confianza para discutir aspectos claves de la acción pedagógica. En este plano también se encuentra la distribución del liderazgo, relacionado íntimamente con los espacios y niveles de participación de los docentes que se dan en la institución, podemos decir que se ha logrado avanzar desde espacios ocasionales de consulta y socialización de propuestas a niveles de participación más protagónicos, que posibilitan el involucramiento docente en procesos de innovación donde ellos son parte central de su implementación.

En cuanto al trabajo colaborativo, es claro que deben asegurarse aspectos estructurales, como condiciones laborales que consideren tiempos de reunión entre profesores, y una vez cumplida esta condición básica, asegurar que estos espacios de reunión se organicen y planifiquen para asegurar reflexión e intercambio profesional que repercuta realmente en la mejora de las prácticas pedagógicas.

El resto de las instituciones analizadas durante la primera etapa del estudio comparten elementos opuestos a la sostenibilidad, lo que se explica, en parte, en dos de ellas, por el hecho de tener en común un sostenedor que incide negativamente en cuanto a disponibilidad de recursos y muestra despreocupación por la continuidad de equipos directivos y docentes. Ante esto cabe preguntarse por las razones por las cuales los distintos niveles institucionales de los cuales depende un liceo, no generan sinergia entre ellos. Por un lado, a nivel ministerial, se invierten recursos para financiar asistencias técnicas, pero por otro se evidencia nulo seguimiento ni medidas tendientes a preservar los logros de dichas inversiones en proyectos y capacitación, desde el nivel de los DAEM, que en este caso constituye un particular ejemplo de la deficiente administración y uso de recursos, a lo que se agrega su desvinculación de los objetivos que se intentan cumplir a nivel de los liceos.

Existe otra serie de elementos que condicionan negativamente la sostenibilidad de la mejora educativa, que bien podrían considerarse en futuros procesos de asesoría técnica. En primer término, es necesario asegurar la apropiación de la propuesta. Para ello la evidencia empírica resalta la importancia del empalme inicial entre asesores y asesorados, un énfasis práctico, el uso del modelaje, a lo que podríamos agregar la necesidad de intensificar el seguimiento de la implementación de cambios, lo que eventualmente puede dejar espacios para ajustes y para flexibilizar las propuestas iniciales. Por otra parte, el 
deficiente involucramiento colectivo pudiera asociarse, entre otros, al sesgo presente en las asesorías de centrar el trabajo pedagógico en las asignaturas de Matemática y Lenguaje y comunicación, lo que se explica en gran medida por la presión que viven estas instituciones por mejorar sus resultados en las mediciones externas. En este sentido, bien vale la pena evaluar la necesidad de ampliar la mirada hacia el conjunto de los docentes en la línea de la reflexión sobre criterios de buena práctica pedagógica que resultan transversales a todas las asignaturas. En esta misma línea, sería fundamental abordar problemáticas presentes en estas instituciones como la visión negativa sobre el potencial de aprendizaje de los estudiantes. Ante esto cabe preguntarse ¿Podrían las asesorías técnicas colaborar en que los docentes desarrollen mayores habilidades para establecer una relación pedagógica positiva con sus estudiantes? ¿El incremento en la incorporación de la cultura juvenil, podría hacer más pertinente su labor con los jóvenes? ¿Podría incluirse en este trabajo de mejora un mejor diagnóstico de la realidad familiar de sus estudiantes para proponer instancias de participación que logren mayor adhesión con la labor educativa del liceo? Creemos que la respuesta a estas interrogantes es afirmativa, pero que ello requiere apuestas de largo plazo en el plano formativo, desde una lógica de educación integral.

\section{CONCLUSIÓN}

Parte de los logros identificados en este estudio se relacionan con aspectos de la cultura organizacional dentro de esta, la cercanía en las relaciones interpersonales, el vínculo positivo a nivel de la comunidad. Se habla de valoración y respeto profesional entre los diferentes miembros del equipo directivo. Relaciones positivas tanto con los profesores como con los estudiantes. En este caso, lo que constituye un logro es, a su vez, una condición de la mejora sostenible.

Lo mismo ocurre en términos de la gestión directiva, la cual al enfatizar metas que dirigen los esfuerzos, que además son monitoreadas en su implementación a través de una acción coordinada y sistemática, constituye un proceder propio de instituciones que asumen procesos de mejora permanentes. Cabe destacar que el monitoreo en relación a la labor pedagógica se presenta más que como una supervisión que como un acompañamiento, lo que tiene una estrecha relación con el cuidado por el clima relacional al interior de la institución.

Bien vale la pena, por tanto, que las asesorías técnicas busquen generar estas condiciones institucionales como elementos sobre los cuales se puedan construir innovaciones, lo que puede tener que ver con enfocarse en procesos de largo alcance a nivel institucional, más que en resultados de aprendizaje que se persigan ciegamente y desde una mirada cortoplacista, que seguramente responde a la presión institucional por demostrar avances en las mediciones externas propias de nuestro sistema educacional.

\section{REFERENCIAS BIBLIOGRÁFICAS}

Anderson, S., \& Carrasco, A. (2013). Nuevas perspectivas para la mejora escolar en tiempos de crecientes políticas de estandarización y rendición de cuentas: evidencia proveniente del Congreso ICSEI. Pensamiento Educativo. Revista de Investigación Educacional Latinoamericana, 5(2), 1-4. 
Bellei, C., Osses, A., \& Valenzuela, J. (2010). Asistencia técnica educativa: de la intuición a la evidencia. Santiago: Ocho Libros.

Cremers, B., Stoll, L., Reezigt, G., \& ESI-Team. (2007). Effective School Improvement-Ingredients for Success: the Results of an International Comparative Study of Best Practice Case Studies. International Handbook of School Effectiveness and Improvement (pp. 825-838). Dordrecht: Springer. Recuperado desde: http://www.rug.nl/staff/b.p.m.creemers/effective_school_ improvement_ingredients_for_success.pdf

Espínola, V., Chaparro, M., Fuenzalida, A., Silva, M., \& Zárate, G. (2008). Estructura organizacional de la administración educativa municipal para asegurar la efectividad del apoyo y seguimiento a Escuelas vulnerables. Informe Proyecto Fonide, $\mathrm{N}^{\circ} 39$. Recuperado desde: www.mineduc.cl

Espínola, V., \& Silva, M. (2009). Competencias del sostenedor para una efectiva gestión del mejoramiento educativo en el nivel local: una propuesta. Foco Educación, CPCE / Expansiva UDP, 1-31. Recuperado desde: http://www2.expansiva.cl/media/en_foco_edu/ documentos/11122009123954.pdf

González, C., \& Bellei, C. (2013). Sostenibilidad del mejoramiento escolar impulsado por programas de asistencia técnica educativa. Perspectiva educacional, 52(1), 31-67.

Hargreaves, A., \& Fink, D. (2006). Estrategias de cambio y mejora en educación caracterizadas por su relevancia, difusión y continuidad en el tiempo. Revista de Educación, 339, 43-58.

Hernández, R., Murillo, F.J., \& Martínez, C. (2014). Factores de ineficacia escolar. Revista Iberoamericana sobre Calidad, Eficacia y Cambio en Educación, 12(1), 103-118.

Hernández, V., \& Santana, P. (2010). Procesos implicados en la mejora escolar: las condiciones institucionales. Revista Iberoamericana sobre Calidad, Eficacia y Cambio en Educación, 8(5), 44-58.

Latorre, A., Rincón, D., \& Arnal, J. (1996). Bases metodológicas de la investigación educativa. Barcelona: GR92.

López, J. (2010). Sostenibilidad de la innovación en los centros escolares: sus bases institucionales. Profesorado. Revista de currículum y formación del profesorado, 14(1), 9-28. Recuperado desde: http://www.ugr.es/ recfpro/rev141ART1.pdf

Marchant, J., Salín, A., \& Williams, J. (2009). Experiencias de intervención institucional en escuelas vulnerables de Latinoamérica: una revisión desde la mejora de la calidad. Revista Iberoamericana sobre Calidad, Eficacia y Cambio en Educación, 7(3), 153-170.

Miles, M., \& Huberman, M. (1994). Qualitative Date Analysis: a source book new methods. Thousand Oaks, CA.: Sage.

Muñoz, G., \& Marfán, J. (2011). Competencias y formación para un liderazgo escolar efectivo en Chile. Pensamiento Educativo. Revista de Investigación Educacional Latinoamericana, 48(1), 63-80.

Murillo, J., \& Krichesky, G. (2012). El proceso del cambio escolar. Una guía para impulsar y sostener la mejora de las escuelas. Revista Iberoamericana sobre Calidad, Eficacia y Cambio en Educación, 10(1), 26-43.

Osses, A., \& Raczinski, D. (2010). Asistencia Técnica Educativa ¿Aporte al Mejoramiento Escolar? Ponencia Seminario Asistencia Técnica Educativa: De la Intuición a la Evidencia. Santiago: CIAE- Universidad de Chile.

Ríos, D., Maturana, D., Almonacid, C., Shink, H., \& Jaramillo, I. (2010). Innovaciones en centros educativos vulnerables: liceos que optimizan la gestión para mejorar los aprendizajes de sus estudiantes. Revista Iberoamericana de Educación, 53(6), 1-12.

Ríos, D., \& Villalobos, P. (2012). Asesoría para el mejoramiento educativo de liceos de alta vulnerabilidad social y de bajo rendimiento académico: reflexiones sobre los facilitadores de la innovación. Perfiles educativos, XXXIV(138), 46-61.

Rodríguez, G., Gil, J., \& García, E. (1996). Metodología de la investigación cualitativa. Archidona: Aljibe. 
Sepúlveda, C., \& Murillo, J. (2012). El origen de los procesos de mejora de la escuela. Un estudio cualitativo en 5 escuelas chilenas. Revista Iberoamericana sobre Calidad, Eficacia y Cambio en Educación, 10(3), 6-24.

Valenzuela, J., \& Allende, C. (2014). Trayectorias de mejoramiento en el sistema educacional chileno. Apuntes sobre mejoramiento escolar. UNICEF-CIAE, 1, 1-15. 